\title{
Real-World Virological Efficacy and Safety of Ledipasvir and Sofosbuvir in Patients with Chronic Hepatitis C Virus Genotype 2 Infection: A Multicenter Study
}

Toshifumi Tada (1) - Takashi Kumada - Hiroaki Okushin - Joji Tani - Koichi Takaguchi $\cdot$ Akemi Tsutsui • Hidenori Toyoda Satoshi Yasuda Kazufumi Dohmen · Atsushi Hiraoka Kojiro Michitaka Kazuhiro Nouso · Kazuya Kariyama · Soo Ryang Kim · Soo Ki Kim · Shinichi Fujioka · Shigeru Mikami · Yuto Watanabe · Tsutomu Tamai $\cdot$ Masanori Atsukawa Norio Itokawa $\cdot$ Hironori Tanaka $\cdot$ Kunihiko Tsuji $\cdot$ Toru Ishikawa Michitaka Imai $\cdot$ Ei Itobayashi $\cdot$ Hiroshi Shibata $\cdot$ Noritomo Shimada

Received: September 26, 2020 / Accepted: October 20, 2020 / Published online: November 3, 2020 (C) The Author(s) 2020

\section{ABSTRACT}

Introduction: The real-world virological efficacy and safety of interferon-free direct-acting antiviral (DAA) therapy with ledipasvir (LDV) plus sofosbuvir (SOF) were assessed in patients

T. Tada $(\bowtie) \cdot$ H. Okushin

Department of Internal Medicine, Japanese Red Cross Society Himeji Hospital, Himeji, Japan

e-mail: tadat0627@gmail.com

T. Kumada

Faculty of Nursing, Gifu Kyoritsu University, Ogaki, Japan

J. Tani

Department of Internal Medicine, Yashima General Hospital, Takamatsu, Japan

K. Takaguchi · A. Tsutsui

Department of Hepatology, Kagawa Prefectural

Central Hospital, Takamatsu, Japan

H. Toyoda $\cdot$ S. Yasuda

Department of Gastroenterology and Hepatology,

Ogaki Municipal Hospital, Ogaki, Gifu, Japan

K. Dohmen

Department of Internal Medicine, Chihaya Hospital, Fukuoka, Japan

A. Hiraoka $\cdot$ K. Michitaka

Gastroenterology Center, Ehime Prefectural Central

Hospital, Matsuyama, Japan who were chronically infected with hepatitis C virus (HCV) genotype 2 .

Methods: A total of 126 patients with chronic hepatitis $\mathrm{C}$ due to $\mathrm{HCV}$ genotype 2 infection who were treated with the LDV/SOF regimen were enrolled. The sustained virological response (SVR) rate and safety were analyzed. SVR was assessed in the intention-to-treat (ITT)

K. Nouso · K. Kariyama

Department of Gastroenterology, Okayama City

Hospital, Okayama, Japan

S. R. Kim · S. K. Kim

Department of Gastroenterology, Kobe Asahi Hospital, Kobe, Japan

\section{S. Fujioka}

Department of Gastroenterology, Okayama Saiseikai General Hospital, Okayama, Japan

S. Mikami · Y. Watanabe

Division of Gastroenterology, Department of Internal Medicine, Kikkoman General Hospital, Noda, Japan

\section{T. Tamai}

Department of Gastroenterology, Kagoshima City Hospital, Kagoshima, Japan

M. Atsukawa · N. Itokawa

Division of Gastroenterology and Hepatology, Department of Internal Medicine, Nippon Medical School, Tokyo, Japan 
population as well as in the modified intentionto-treat (mITT) population, which excluded patients with non-virological failure, including those who dropped out before the SVR assessment.

Results: The overall SVR rates of the ITT and mITT populations were $87.3 \%$ (95\% confidence interval [CI] 80.2-92.6) (110/126) and 97.3\% (95\% CI 92.4-99.4) (110/113), respectively. In the mITT population, the percentages of patients with undetectable HCV RNA at 4, 8, and 12 weeks after the start of therapy were 92.9\% (95\% CI 86.5-96.9) (105/113), 99.1\% (95\% CI 95.2-100.0) (112/113), and $100.0 \%$ (95\% CI 97.4-100.0) (113/113), respectively. Subgroup analyses of the mITT population showed no significant differences in SVR rates according to age, sex, HCV genotype (subtype), history of interferon-based therapy, baseline FIB-4 index, or baseline estimated glomerular filtration rate. In all subpopulations, the SVR rates were $>90 \%$. There were no severe adverse events associated with the treatment.

Conclusion: The LDV/SOF regimen showed high virological efficacy and acceptable safety in patients with HCV genotype 2 infection.

Trial Registration: UMIN registration no. 000038604.

H. Tanaka

Department of Gastroenterology and Hepatology,

Takarazuka Municipal Hospital, Takarazuka, Japan

K. Tsuji

Center of Gastroenterology, Teine Keijinkai

Hospital, Sapporo, Japan

T. Ishikawa $\cdot$ M. Imai

Department of Gastroenterology, Saiseikai Niigata

Hospital, Niigata, Japan

E. Itobayashi

Department of Gastroenterology, Asahi General

Hospital, Asahi, Japan

H. Shibata

Department of Gastroenterology, Tokushima

Prefectural Central Hospital, Tokushima, Japan

N. Shimada

Division of Gastroenterology and Hepatology,

Otakanomori Hospital, Kashiwa, Japan
Keywords: Genotype 2; Hepatitis C virus; Ledipasvir; Sofosbuvir; Sustained virological response

\section{Key Summary Points}

\section{Why carry out this study?}

In a Japanese randomized controlled trial, LDV/SOF treatment in patients with $\mathrm{HCV}$ genotype 2 resulted in high SVR rates $(96 \%)$ that were equivalent to those of SOF plus ribavirin (95\%).

Real-world clinical data on LDV/SOF treatment for patients with HCV genotype 2 are limited.

To investigate the real-world outcomes of LDV/SOF treatment for patients with HCV genotype 2 in a multicenter study conducted throughout Japan.

\section{What was learned from the study?}

The overall SVR rates of the ITT and mITT populations were $87.3 \%(110 / 126)$ and $97.3 \%$ (110/113), respectively.

There were no severe adverse events associated with the treatment.

The LDV/SOF regimen showed high virological efficacy and acceptable safety in patients with HCV genotype 2 .

\section{DIGITAL FEATURES}

This article is published with digital features, including a summary slide, to facilitate understanding of the article. To view digital features for this article go to https://doi.org/10.6084/ m9.figshare.13118183.

\section{INTRODUCTION}

Hepatitis C virus (HCV) infection affected 71 million people globally in 2015 [1]. It is a major 
risk factor for cirrhosis, hepatocellular carcinoma (HCC), and liver-related death. In Japan, 1.0-1.5 million individuals are infected with $\mathrm{HCV}$, and approximately $55 \%$ of HCC is associated with chronic HCV infection [2]. Anti$\mathrm{HCV}$ therapy to eradicate $\mathrm{HCV}$ is an essential treatment for chronic $\mathrm{HCV}$ infection, as it reportedly prevents the progression of liver fibrosis [3-5] and the development of HCC [6-10] and also reduces all-cause mortality, including mortality due to non-liver-related diseases [11, 12].

Among the six HCV genotypes, HCV genotype 2 accounts for approximately $13 \%$ of infections globally [13]. This HCV genotype is common in Latin America, sub-Saharan Africa, and East Asia. In many countries in these regions, official approval for new direct-acting antivirals (DAAs) has been delayed because DAA treatment is costly and market access and governmental restrictions differ between certain countries. The current guidelines of the American Association for the Study of Liver Diseases [14] and the European Association for the Study of the Liver [15] recommend the following interferon-free DAA regimens for patients with HCV genotype 2 infection: sofosbuvir (SOF) plus velpatasvir for 12 weeks or glecaprevir plus pibrentasvir for 8-12 weeks.

Until recently, approximately $70 \%$ of patients with HCV infection in Japan had genotype $1 \mathrm{~b}$ and the remainder had genotype 2 [16]. Lately, however, the proportion of HCV genotype $1 \mathrm{~b}$ has decreased and is now $<50 \%$ in individuals born after 1970. By contrast, the percentage of HCV genotype 2 infection, especially $2 b$, has increased in younger generations. The current guidelines of the Japan Society of Hepatology [17] recommend the following interferon-free DAA regimens for non-decompensated cirrhotic patients with HCV genotype 2 infection: SOF plus ribavirin for 12 weeks, glecaprevir plus pibrentasvir for 8-12 weeks, or ledipasvir (LDV) plus SOF for 12 weeks. LDV is an HCV NS5A inhibitor, and SOF is a potent NS5B nucleotide polymerase inhibitor with pan-genotypic activity and a high barrier to resistance. In a Japanese randomized controlled trial, LDV/SOF treatment in patients with HCV genotype 2 infection resulted in high sustained virological response (SVR) rates (96\%) that were equivalent to those of SOF plus ribavirin (95\%) [18]. However, real-world clinical data on LDV/ SOF treatment for patients with HCV genotype 2 infection are limited. Therefore, it is necessary to clarify the real-world clinical outcomes of LDV/SOF treatment for patients with $\mathrm{HCV}$ genotype 2 infection in Japan.

In this study, we analyzed the real-world outcomes of this treatment approach for patients with HCV genotype 2 infection in a multicenter study conducted throughout Japan.

\section{METHODS}

\section{Patients}

This prospective cohort study was conducted to characterize the prevalence of nonhypervascular hypointense nodules (NHHNs) during the hepatobiliary phase of EOB-MRI and the incidence of hyper-vascularization among patients in whom HCV was eradicated with DAA therapy. The study was registered to the UMIN Clinical Trial Registry (UMIN000017020). The study was conducted after approval by the hospital's Institutional Review Board. It was carried out in compliance with the Helsinki Declaration. Written informed consent was obtained from all participating patients. This study was approved by the ethics committee of Ogaki Municipal Hospital (IRB \#20180927-13k). All documents approved by Ogaki Municipal Hospital (Kumada T) were sent to each institution and approved by each institution under the same IRB name and number. This study was performed in accordance with the 2013 Helsinki Declaration. All patients consented to provide their data. Written informed consent was obtained from each patient before enrollment. All patients consented to provide their data. Consent to participate and allow their data to be published was obtained from each patient before enrollment.

This was a multicenter study conducted at 18 institutions in the Hokkaido, Kanto, Hokushinnestu, Chubu, Kansai, Chugoku, Shikoku, and Kyusyu areas of Japan. A total of 126 patients with chronic hepatitis $\mathrm{C}$ due to HCV genotype 2 
infection who underwent DAA treatment with the LDV/SOF regimen between September 2018 and September 2019 were enrolled. Persistent infection with HCV genotype 2 was proved in all patients by both positive serum HCV antibody titers (ARCHITECT Anti-HCV; Abbott Laboratories, Abbott Park, IL, USA) and the presence of serum HCV RNA determined using a real-time PCR-based method (COBAS AmpliPrep/COBAS TaqMan HCV Test version 2; Roche Molecular Systems, Pleasanton, CA, USA; lower limit of quantification, $1.6 \log _{10} \mathrm{IU} / \mathrm{ml}$; lower limit of detection, $\left.1.2 \log _{10} \mathrm{IU} / \mathrm{ml}\right)$. HCV genotype was evaluated by PCR with genotypespecific primers to amplify the core gene sequences [19]. In some institutions, only the HCV serotype could be determined by a serotyping assay based on the type-specific antibodies because of insurance coverage. Patients with severe chronic renal failure were excluded. Patients with decompensated cirrhosis were also excluded because this DAA regimen is contraindicated for this population in Japan.

\section{Treatment and Follow-Up}

Patients received fixed doses of LDV (90 mg) and SOF (400 mg) (Harvoni; Gilead Sciences, Tokyo, Japan) once daily on an outpatient basis. The duration of the treatment regimen was scheduled for 12 weeks. Patients were asked to visit the clinic for monitoring of treatment response every 2 weeks throughout the treatment period and every 4 weeks during followup, which lasted until 12 weeks after the end of treatment. Laboratory data, specifically complete blood count and serum levels of alanine aminotransferase, aspartate aminotransferase, albumin, total bilirubin, and creatinine, were measured before and every 2-4 weeks after the start of therapy. Serum HCV RNA levels were measured before treatment; at 4, 8, and 12 weeks after the start of therapy while treatment was ongoing; at the end of treatment; and at 12 weeks after the end of treatment. Viral suppression was defined as undetectable serum HCV RNA. Relapse was defined when serum HCV RNA was undetectable at the end of therapy but became detectable between the end of treatment and 12 weeks after the completion of therapy. Breakthrough was defined when HCV RNA became detectable after its initial disappearance during treatment. Non-response was defined when serum HCV RNA levels increased despite their initial decrease during treatment. SVR was defined as undetectable serum HCV RNA at 12 weeks after the end of treatment. In this study, SVR was assessed in the intention-to-treat (ITT) population as well as in the modified intention-to-treat (mITT) population, which excluded patients with non-virological failure, including those who dropped out before the SVR assessment. Potential adverse events, including laboratory value abnormalities that emerged after the start of therapy, were monitored by attending physicians at every patient visit. Treatment discontinuation was determined based on attending physicians' evaluations.

\section{Assessment of Advanced Liver Fibrosis and Renal Dysfunction}

Liver fibrosis was assessed based on pretreatment laboratory data using the FIB-4 index, a laboratory liver fibrosis index defined by the following formula [20]: aspartate aminotransferase $[\mathrm{IU} / \mathrm{l}] \times$ age [years]/platelet count $\left[10^{9} /\right.$ 1] $\times$ alanine aminotransferase $[\mathrm{IU} / \mathrm{l}]^{1 / 2}$. Patients were defined as having advanced fibrosis (i.e., F3 or F4 by the METAVIR score [21]) when the FIB-4 index was $\geq 3.25$ [22].

Renal function was assessed by the estimated glomerular filtration rate (eGFR) $(\mathrm{ml} / \mathrm{min} / 1.73$ $\mathrm{m}^{2}$ ) based on pretreatment laboratory data using the following formula [23]: $194 \times$ serum creatinine $\quad(\mathrm{mg} / \mathrm{dl})-1.094 \times$ age $-0.287 \times$ 0.739 (if female). Chronic kidney disease was classified as eGFR $<60 \mathrm{ml} / \mathrm{min} / 1.73 \mathrm{~m}^{2}$ [24]. In addition, severe chronic renal failure was classified as eGFR $<30 \mathrm{ml} / \mathrm{min} / 1.73 \mathrm{~m}^{2}$ [24].

\section{Statistical Analysis}

Continuous variables are expressed as medians (the first-third quartiles). For comparisons of baseline characteristics and SVR rates between 
Table 1 Patient characteristics $(n=126)$

Age (years)*

Sex (female/male)

History of interferon-based therapy (no/yes)

History of curatively treated HCC (no/yes)

Aspartate aminotransferase (IU/l)*

Alanine aminotransferase (IU/1)*

$\gamma$-glutamyl transpeptidase $(\mathrm{IU} / \mathrm{l})^{*}$

Albumin $(\mathrm{g} / \mathrm{dl})^{*}$

Total bilirubin $(\mathrm{mg} / \mathrm{dl})^{*}$

Creatinine $(\mathrm{mg} / \mathrm{dl})^{*}$

Prothrombin time $(\%)^{*}$

Platelet count $\left(\times 10^{4} / \mathrm{mm}^{3}\right)^{*}$

Hemoglobin $(\mathrm{g} / \mathrm{dl})^{*}$

Fasting plasma glucose $(\mathrm{mg} / \mathrm{dl})^{*}$

HCV genotype (2a/2b/2 [un-subtyped])

HCV RNA $\left(\log _{10} \mathrm{IU} / \mathrm{ml}\right)^{*}$

FIB-4 index*

eGFR $\left(\mathrm{ml} / \mathrm{min} / 1.73 \mathrm{~m}^{2}\right)^{*}$
$60.5(47.0-74.0)$

$65 / 61$

$116 / 10$

$121 / 5$

$34(26-54)$

$36(21-69)$

$38(20-64)$

$4.1(3.8-4.4)$

$0.6(0.5-0.8)$

$0.72(0.60-0.83)$

$103(91-112)$

$19.8(15.2-26.1)$

$13.7(12.8-14.8)$

$102(91-113)$

$80 / 40 / 6$

$6.1(4.9-6.6)$

$1.83(0.95-3.19)$

$62.4(41.0-87.3)$

$H C V$ hepatitis $\mathrm{C}$ virus, $H C C$ hepatocellular carcinoma, $e G F R$ estimated glomerular filtration rate

*Values are expressed as medians (first to third quartiles)

patient subgroups, differences in categorical variables were analyzed using Fisher's exact test.

All statistical analyses were performed with EZR (Saitama Medical Center, Jichi Medical University, Saitama, Japan), which is a graphical user interface for R (The R Foundation for Statistical Computing, Vienna, Austria) [25]. More precisely, it is a modified version of the $R$ commander designed to add statistical functions frequently used in biostatistics. Statistical significance was defined as $p<0.05$.

\section{RESULTS}

\section{Patient Characteristics}

Table 1 shows the characteristics of the study patients at the start of DAA therapy. There were
65 females (51.6\%) and 61 males (48.4\%), with a median (interquartile range) age of 60.5 (47.0-74.0) years.

The age was $\geq 70$ years in 46 patients (36.5\%), including 18 patients (14.3\%) who were $\geq 80$ years old. Ten patients $(7.9 \%)$ had a history of interferon-based therapy, and one patient $(0.8 \%)$ had a history of interferon-free DAA therapy. The FIB-4 index at baseline was $>3.25$ in 31 patients (24.6\%). Five patients $(4.0 \%)$ had a history of curatively treated HCC. Renal dysfunction with eGFR $<60 \mathrm{ml} / \mathrm{min} / 1.73$ $\mathrm{m}^{2}$ was observed in 59 patients $(46.8 \%)$. Among patients who underwent HCV subtyping, there were $80(63.5 \%)$ and $40(31.5 \%)$ patients with genotype $2 \mathrm{a}$ and $2 \mathrm{~b}$, respectively. 


\section{Virological Treatment Outcomes in the ITT and mITT Populations}

The overall SVR rates in the ITT and mITT populations were $87.3 \% \quad(95 \%$ confidence interval [CI] 80.2-92.6) (110/126) and 97.3\% (95\% CI 92.4-99.4) (110/113), respectively.

The percentages of the mITT population with undetectable $\mathrm{HCV}$ RNA at 4, 8, and 12 weeks after the start of therapy were $92.9 \%$ (95\% CI 86.5-96.9) (105/113), 99.1\% (95\% CI
95.2-100.0) (112/113), and 100.0\% (95\% CI 97.4-100.0) (113/113), respectively.

\section{Virological Treatment Outcomes in the ITT Population Subgroups}

Figure 1 shows SVR rates based on patient age, sex, HCV genotype (subtype), history of interferon-based therapy, baseline FIB-4 index, and baseline eGFR in the ITT population. The SVR rates in patients with baseline FIB-4 index

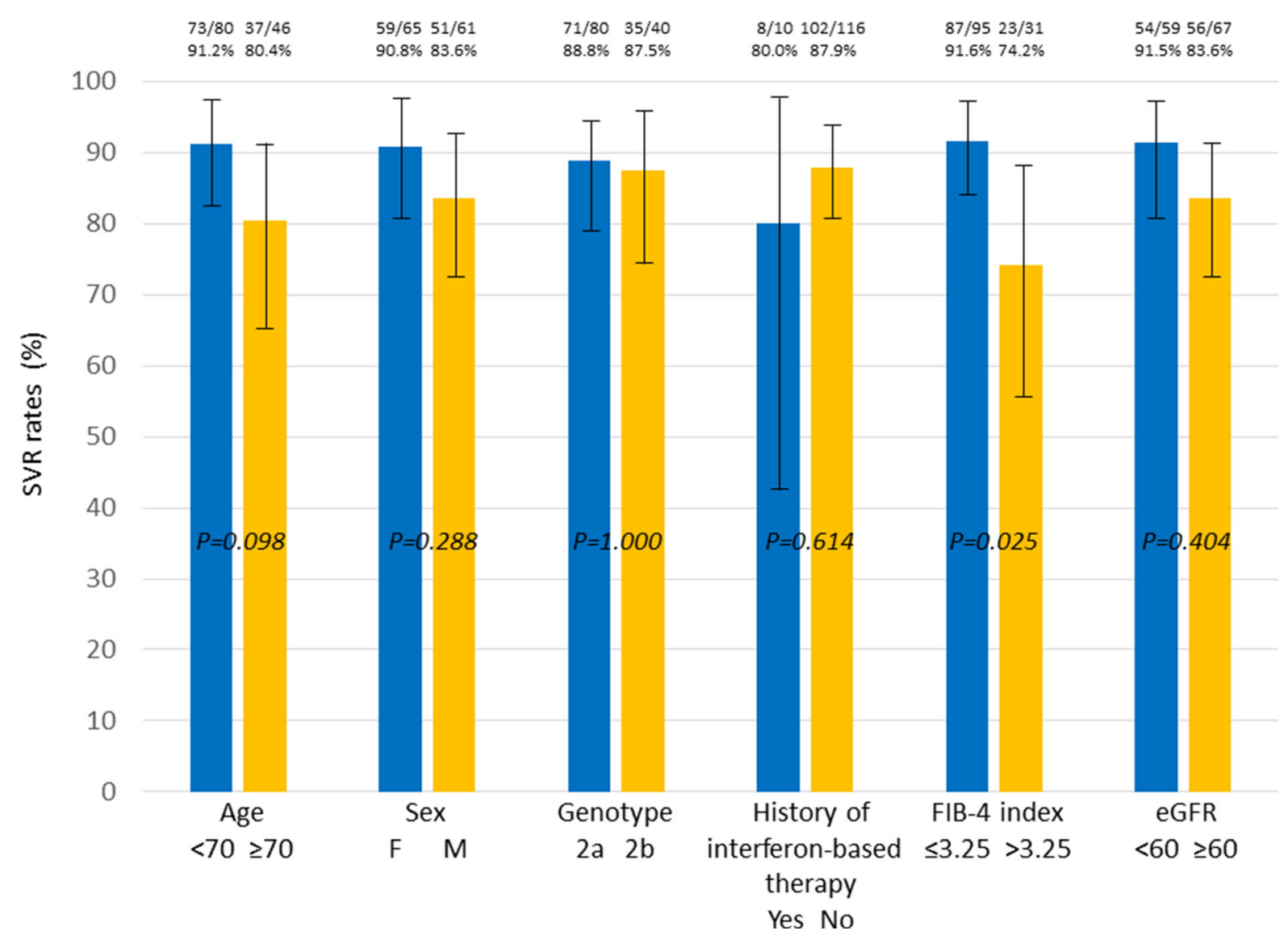

Fig. 1 SVR rates in ITT population subgroups. The SVR rates in the ITT population were stratified by subgroups, as follows: patient age ( $<70$ years $>70$ years), $91.2 \%$ ( $95 \%$ CI 82.8-96.4) (73/80)/80.4\% (95\% CI 66.1-90.6) (37/ 46) $(p=0.098)$; sex (female/male), $90.8 \% \quad(95 \% \mathrm{CI}$ 81.0-96.5) (59/65)/83.6\% (95\% CI 71.9-91.8) (51/61) $(p=0.288) ; \mathrm{HCV}$ genotype $(2 \mathrm{a} / 2 \mathrm{~b}), 88.8 \%$ (95\% CI 79.7-94.7) (71/80)/87.5\% (95\% CI 73.2-95.8) (35/40) $(p=1.000)$; history of interferon-based therapy (yes/no), $80.0 \% \quad(95 \%$ CI $44.4-97.5) \quad(8 / 10) / 87.9 \% \quad(95 \% \quad$ CI
80.6-93.2) (102/116) $(p=0.614)$; baseline FIB-4 index $(\leq 3.25 />3.25), 91.6 \%$ (95\% CI 84.1-96.3) (87/95)/ $74.2 \%$ (95\% CI 55.4-88.1) (23/31) ( $p=0.025)$; and baseline eGFR $\left(<60 / \geq 60 \mathrm{ml} / \mathrm{min} / 1.73 \mathrm{~m}^{2}\right), \quad 91.5 \%$ (95\% CI 81.3-97.2) (54/59)/83.6\% (95\% CI 72.5-91.5) $(56 / 67)(p=0.404)$, respectively. The error bars indicate the lower and upper values of the $95 \%$ CI of each SVR rate. $S V R$ sustained virological response, ITT intention to treat, $H C V$ hepatitis $\mathrm{C}$ virus, $C I$ confidence interval 
$(\leq 3.25 />3.25)$ were $91.6 \%$ (95\% CI 84.1-96.3) (87/95)/74.2\% (95\% CI 55.4-88.1) (23/31). The SVR rate was significantly lower in patients with a baseline FIB-4 index $\geq 3.25(p=0.025)$, but did not differ significantly according to any of the other parameters.

\section{Reasons for Lack of Inclusion in the mITT Population}

Thirteen patients were excluded from the ITT population. There were $1,1,1,1$, and 3 patients who self-discontinued their DAA administration at $1,2,4,6$, and 8 weeks after the start of treatment, respectively. In addition, there were 1,1 , and 3 patients who self-discontinued their visits at 0,4 , and 8 weeks after the end of treatment, respectively. One patient who discontinued DAA administration because of worsening of current disease (worsening of Parkinson's symptoms).

\section{Virological Treatment Outcomes in mITT Population Subgroups}

Figure 2 shows SVR rates based on patient age, sex, HCV genotype (subtype), history of interferon-based therapy, baseline FIB-4 index, and baseline eGFR in the mITT population. There were no significant differences in SVR rates according to these parameters.

Table 2 lists the characteristics of three patients who failed to achieve SVR in the mITT population. All three patients relapsed after the end of therapy. Of these, two patients were infected with genotype $2 \mathrm{~b}$ HCV and had a baseline FIB- 4 index of $\geq 3.25$.

\section{Safety}

Two patients had adverse events during the DAA treatment. In one patient (84-year-old female; genotype 2a) with Parkinson's disease, the DAA treatment was discontinued at 1 week after the start of therapy because of worsening of Parkinson's symptoms. These symptoms improved after the DAA treatment was stopped. However, the causal relation of the treatment and this adverse effect was unclear. Another patient (50-year-old female; genotype 2a) developed oral ulcers at 2 weeks after the start of therapy, but they were mild and she was able to continue treatment. These oral ulcers were considered as an adverse event related to LDV/ SOF treatment by the attending physician.

\section{DISCUSSION}

This multicenter clinical study conducted throughout Japan enrolled a relatively large number of patients with $\mathrm{HCV}$ genotype 2 infection and either chronic hepatitis or nondecompensated cirrhosis and showed that LDV/ SOF treatment achieved a high SVR rate of $97.3 \%$ in the mITT population. In this group, $\mathrm{HCV}$ RNA was undetectable in $>90 \%$ of patients at 4 weeks after the start of treatment and in almost all patients (99.1\%) at 8 weeks. Furthermore, subgroup analyses of the mITT population based on patient age, sex, HCV genotype (subtype), history of interferon-based therapy, baseline FIB-4 index, and baseline eGFR showed no significant differences in SVR rates according to these parameters. Notably, high SVR rates $>90 \%$ were obtained in the mITT population even in elderly individuals and those with advanced fibrosis or a history of interferon-based therapy. Regarding safety, only one elderly patient discontinued this DAA treatment because of exacerbation of Parkinson's disease symptoms. As a result, there were only one dropout patient and one patient with a mild adverse event (oral ulcers), respectively, associated with this DAA treatment. Therefore, it is considered that the LDV/SOF treatment showed excellent efficacy and acceptable safety in patients with HCV genotype 2 infection.

Genotype $2 \mathrm{HCV}$ has previously shown high rates of response to combination therapy with SOF plus ribavirin, a broad-spectrum antiviral. In three registered studies of SOF plus ribavirin, 12 weeks of treatment resulted in an SVR rate of $94 \%$ among patients with chronic HCV genotype 2 infection [26-28]. Compared with SOF, which has a favorable safety profile and is well tolerated [29], ribavirin has poorer tolerability, mostly because it can cause hemolytic anemia. $\mathrm{HCV}$ patients with ribavirin-associated anemia 


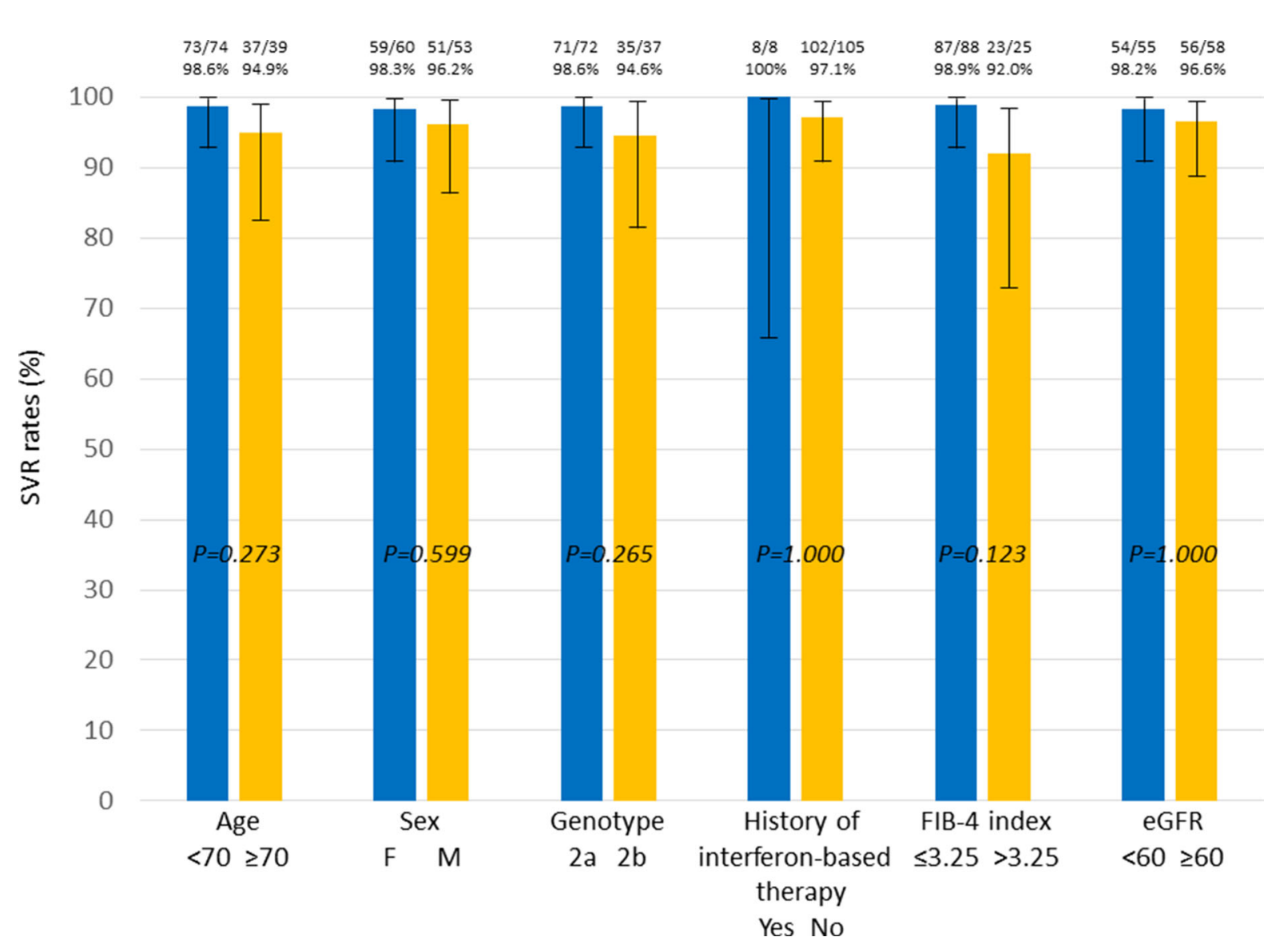

Fig. 2 SVR rates in mITT population subgroups. The SVR rates in the mITT population were stratified by subgroups, as follows: patient age ( $<70$ years $/ \geq 70$ years), 98.6\% (95\% CI 92.7-100.0) (73/74)/94.9\% (95\% CI 82.7-99.4) (37/39) ( $p=0.273)$; sex (female/male), $98.3 \%$ (95\% CI 91.1-100.0) (59/60)/96.2\% (95\% CI 87.0-99.5) $(51 / 53)(p=0.599)$; HCV genotype $(2 \mathrm{a} / 2 \mathrm{~b}), 98.6 \%$ ( $95 \%$ CI 92.5-100.0) (71/72)/94.6\% (95\% CI 81.8-99.3) (35/ 37) $(p=0.265)$; history of interferon-based therapy (yes/ no), $100.0 \%$ (95\% CI 68.8-100.0) (8/8)/97.1\% (95\% CI

have significantly increased resource utilization and medical costs [30].

LDV/SOF has been investigated for the treatment of patients with HCV genotype 2 infection in phase 2 and 3 trials in the AsiaPacific region [18, 31, 32]. Gane et al. [31] reported in their phase 2 trial that the SVR rates at 8 weeks and 12 weeks after the start of LDV/ SOF treatment were 74\% (20/27) and 96\% (25/ 26), respectively, in patients with HCV genotype 2, indicating a significant difference between time points $(p<0.001)$. In a phase 3
91.9-99.4) $(102 / 105)(p=1.000)$; baseline FIB-4 index $(\leq 3.25 />3.25), 98.9 \%$ (95\% CI $93.8-100.0)(87 / 88) /$ 92.0\% (95\% CI 74.0-99.0) (23/25) $(p=0.123)$; and baseline eGFR $\left(<60 / \geq 60 \mathrm{ml} / \mathrm{min} / 1.73 \mathrm{~m}^{2}\right), \quad 98.2 \%$ $\begin{array}{llllll}(95 \% & \text { CI } & 90.3-100.0) & (54 / 55) / 96.6 \% & (95 \% & \text { CI }\end{array}$ 88.1-99.6) (56/58) ( $p=1.000)$, respectively. The error bars indicate the lower and upper values of the $95 \%$ CI of each SVR rate. $S V R$ sustained virological response, $m I T T$ modified intention to treat, $H C V$ hepatitis $\mathrm{C}$ virus, $C I$ confidence interval

trial conducted in a Japanese cohort with $\mathrm{HCV}$ genotype 2 infection, Asahina et al. [18] showed that SVR rates were $96 \%(102 / 106)$ with LDV/ SOF treatment and 95\% (103/108) with SOF plus ribavirin treatment, indicating that $\mathrm{LDV} /$ SOF was non-inferior to SOF plus ribavirin. However, limited real-world clinical data have been reported to date on treatment outcomes with LDV/SOF in patients with HCV genotype 2 infection. There were no differences in patient characteristics such as age and sex between the phase 3 trial from Japan and this study cohort. 
Table 2 Patients who failed to achieve SVR $(n=3)$

\begin{tabular}{llll}
\hline No. & $\mathbf{1}$ & $\mathbf{2}$ & $\mathbf{3}$ \\
\hline Age & 83 & 69 & 71 \\
Sex & Male & Female & Female \\
History of interferon-based therapy & No & No & No \\
History of curatively treated HCC & No & No & Yes \\
HCV genotype & $2 \mathrm{a}$ & $2 \mathrm{~b}$ & $2 \mathrm{~b}$ \\
HCV RNA $\left(\log _{10} \mathrm{IU} / \mathrm{ml}\right)$ & 6.5 & 6.8 & 6.5 \\
FIB-4 index & 2.12 & 6.35 & 10.24 \\
eGFR $\left(\mathrm{ml} / \mathrm{min} / 1.73 \mathrm{~m}^{2}\right)$ & 71.9 & 44.4 & 85.0 \\
Undetectable HCV RNA after the start of therapy & 8 weeks & 8 weeks & 4 weeks \\
Detectable HCV RNA after the end of therapy & 4 weeks & 4 weeks & 12 weeks \\
Details of SVR & Relapse & Relapse & Relapse \\
\hline
\end{tabular}

$H C C$ hepatocellular carcinoma, $H C V$ hepatitis $\mathrm{C}$ virus, $e G F R$ estimated glomerular filtration rate

Although almost all of the patients in this phase 3 trial visited a few university hospitals in Japan, this study's patients visited not only university hospitals but also community-based general hospitals nationwide. Recently, Liu et al. [33] demonstrated good efficacy and safety in Taiwanese patients with HCV genotype 2 infection treated with SOF-based regimens, and all 39 patients who received LDV/SOF treatment achieved SVR. Additionally, in a recent multicenter study in the Kyusyu area of Japan, Ogawa et al. [34] also reported that LDV/SOF treatment of patients with $\mathrm{HCV}$ genotype 2 infection was effective and safe, with an SVR rate of $96.5 \%$ $(55 / 57)$ in the mITT population. In their ITT population $(n=58)$, there were $35(60.3 \%), 10$ (17.2\%), and $13(22.4 \%)$ patients with $\mathrm{HCV}$ genotype $2 \mathrm{a}, 2 \mathrm{~b}$, and 2 (un-subtyped) infection, respectively. Similar to the studies of Liu et al. [33] and Ogawa et al. [34], almost all patients in our study achieved SVR and there were no serious adverse events or deaths. An advantage of our study relative to that of Ogawa et al. was that it included more patients from throughout Japan. In addition, only $4.8 \%(6 / 126)$ of patients with HCV genotype 2 infection were un-subtyped. Unfortunately, there was no Japanese randomized controlled trial regarding the SOF plus velpatasvir treatment for non-decompensated cirrhotic patients with $\mathrm{HCV}$ genotype 2 infection. Only decompensated cirrhotic patients with $\mathrm{HCV}$ infection were accepted to use the SOF plus velpatasvir treatment based on the results of randomized controlled trial in Japan. Conversely, the LDV/SOF treatment for non-decompensated cirrhotic patients with HCV genotype 2 infection was accepted based on the good results of Japanese randomized controlled trial [18] in the Japanese guidelines.

The main limitation of this study was that it did not assess the relationship between SVR rate and NS5A and NS5B mutations. Generally, LDV at half-maximal effective concentration is much less efficacious against $\mathrm{HCV}$ genotype 2 than genotype 1, especially in patients with NS5A L31 M, despite the fact that SOF demonstrates similar efficacy against genotypes 1 and 2 [35]. However, resistance-associated substitution analysis showed that almost all patients (94-97\%) with subtype $2 \mathrm{a}$ infection and $39-89 \%$ of those with subtype $2 b$ infection originally had NS5A L31 M at baseline $[18,36]$. In addition, the SVR rate in patients with NS5A resistance-associated substitutions was extremely high $(97.6 \%, 165 / 169)$ in clinical trials 
$[18,31,32]$. Therefore, it is considered that NS5A L31 M, and not NS5B, is unassociated with treatment outcome. Further studies assessing NS5A and NS5B mutations should be performed. Another limitation is that 13 patients were excluded from the mITT population, mainly because of self-discontinuation of visits during or after LDV/SOF treatment. This may be related to the social background such as a history of intravenous drug use or a history of tattooing in patients with HCV genotype 2 infection [16, 37]. Additional studies of this treatment with fewer dropouts are warranted. Third, it included a relatively small number of patients. Future studies with more patients are warranted.

\section{CONCLUSION}

An IFN-free DAA regimen with LDV/SOF demonstrated a very high SVR rate and acceptable safety profile in patients infected with HCV genotype 2 infection. In particular, the LDV/ SOF regimen resulted in good SVR rates regardless of patient age, sex, HCV genotype (subtype), history of interferon-based therapy, baseline FIB-4 index, and baseline eGFR. Further studies are warranted to confirm these findings in other populations.

\section{ACKNOWLEDGEMENTS}

We thank the participants of the study.

Funding. This work was supported by Gilead Sciences (commercial research funding). Gilead Sciences also funded the journal's Rapid Service Fees.

Authorship. All named authors meet the International Committee of Medical Journal Editors (ICMJE) criteria for authorship for this article, take responsibility for the integrity of the work as a whole, and have given their approval for this version to be published.

Authorship Contributions. Concept and study design: Takashi Kumada and Toshifumi
Tada. Data acquisition: All authors. Analyses of the data: Takashi Kumada and Toshifumi Tada. Statistics: Toshifumi Tada. Supervise: Takashi Kumada. Preparing the manuscript: Toshifumi Tada. Review and approval: All authors.

Disclosures. Takashi Kumada served as a speaker for Gilead and AbbVie. Hidenori Toyoda has served as a speaker for Gilead, AbbVie, and MSD. Toshifumi Tada, Hiroaki Okushin, Joji Tani, Koichi Takaguchi, Akemi Tsutsui, Satoshi Yasuda, Kazufumi Dohmen, Atsushi Hiraoka, Kojiro Michitaka, Kazuhiro Nouso, Kazuya Kariyama, Soo Ryang Kim, Soo Ki Kim, Shinichi Fujioka, Shigeru Mikami, Yuto Watanabe, Tsutomu Tamai, Masanori Atsukawa, Norio Itokawa, Hironori Tanaka, Kunihiko Tsuji, Toru Ishikawa, Michitaka Imai, Ei Itobayashi, Hiroshi Shibata, and Noritomo Shimada declare that they have no competing interests.

Compliance with Ethics Guidelines. This study was approved by the ethics committee of Ogaki Municipal Hospital (IRB \#20180927-13k). All documents approved by Ogaki Municipal Hospital (Kumada T) were sent to each institution and approved by each institution under the same IRB name and number. This study was performed in accordance with the 2013 Helsinki Declaration. All patients consented to provide their data. Written informed consent was obtained from each patient before enrollment. All patients consented to provide their data. Consent to both participate and allow the data to be published was obtained from each patient before enrollment.

Data Availability. The datasets are available from the corresponding author on reasonable request.

Open Access. This article is licensed under a Creative Commons Attribution-NonCommercial 4.0 International License, which permits any non-commercial use, sharing, adaptation, distribution and reproduction in any medium or format, as long as you give appropriate credit to the original author(s) and the source, provide a link to the Creative Commons licence, and 
indicate if changes were made. The images or other third party material in this article are included in the article's Creative Commons licence, unless indicated otherwise in a credit line to the material. If material is not included in the article's Creative Commons licence and your intended use is not permitted by statutory regulation or exceeds the permitted use, you will need to obtain permission directly from the copyright holder. To view a copy of this licence, visit http://creativecommons.org/licenses/by$\mathrm{nc} / 4.0 /$.

\section{REFERENCES}

1. Polaris Observatory HCV Collaborators. Global prevalence and genotype distribution of hepatitis c virus infection in 2015: a modelling study. Lancet GastroenterolHepatol. 2017;2:161-76.

2. Tateishi R, Uchino $\mathrm{K}$, Fujiwara $\mathrm{N}$, et al. A nationwide survey on non- $\mathrm{B}$, non-C hepatocellular carcinoma in Japan: 2011-2015 update. J Gastroenterol. 2019;54:367-76.

3. Marcellin P, Boyer N, Gervais A, et al. Long-term histologic improvement and loss of detectable intrahepatic HCV RNA in patients with chronic hepatitis $C$ and sustained response to interferon-alpha therapy. Ann Intern Med. 1997;127:875-81.

4. Shiffman ML, Hofmann CM, Thompson EB, et al. Relationship between biochemical, virological, and histological response during interferon treatment of chronic hepatitis C. Hepatology. 1997;26:780-5.

5. Shiratori Y, Imazeki F, Moriyama M, et al. Histologic improvement of fibrosis in patients with hepatitis $\mathrm{C}$ who have sustained response to interferon therapy. Ann Intern Med. 2000;132:517-24.

6. Ikeda K, Saitoh S, Arase Y, et al. Effect of interferon therapy on hepatocellular carcinogenesis in patients with chronic hepatitis type C: a long-term observation study of 1,643 patients using statistical bias correction with proportional hazard analysis. Hepatology. 1999;29:1124-30.

7. Imai Y, Kawata S, Tamura S, et al. Relation of interferon therapy and hepatocellular carcinoma in patients with chronic hepatitis C. Ann Intern Med. 1998;129:94-9.

8. Yoshida H, Shiratori Y, Moriyama M, et al. Interferon therapy reduces the risk for hepatocellular carcinoma: national surveillance program of cirrhotic and noncirrhotic patients with chronic hepatitis $\mathrm{C}$ in Japan. IHIT study group. Inhibition of hepatocarcinogenesis by interferon therapy. Ann Intern Med. 1999;131:174-81.

9. Kasahara A, Hayashi N, Mochizuki K, et al. Risk factors for hepatocellular carcinoma and its incidence after interferon treatment in patients with chronic hepatitis C. Hepatology. 1998;27: 1394-402.

10. Ogawa E, Furusyo N, Kajiwara E, Kyushu University Liver Disease Study (KULDS) Group, et al. Efficacy of pegylated interferon alpha- $2 b$ and ribavirin treatment on the risk of hepatocellular carcinoma in patients with chronic hepatitis C: a prospective, multicenter study. J Hepatol. 2013;58:495-501.

11. van der Meer AJ, Veldt BJ, Feld JJ, et al. Association between sustained virological response and allcause mortality among patients with chronic hepatitis $\mathrm{C}$ and advanced hepatic fibrosis. JAMA. 2012;308:2584-93.

12. Tada $\mathrm{T}$, Kumada $\mathrm{T}$, Toyoda $\mathrm{H}$, et al. Viral eradication reduces all-cause mortality in patients with chronic hepatitis $\mathrm{C}$ virus infection: a propensity score analysis. Liver Int. 2016;36:817-26.

13. Gower E, Estes C, Blach S, Razavi-Shearer K, Razavi H. Global epidemiology and genotype distribution of the hepatitis $\mathrm{c}$ virus infection. J Hepatol. 2014;61:S45-57.

14. AASLD-IDSAHCV Guidance Panel. Hepatitis C guidance 2018 update: AASLD-IDSA recommendations for testing, managing, and treating hepatitis C virus infection. Clin Infect Dis. 2018;67:1477-92.

15. European Association for the Study of the Liver. EASL recommendations on treatment of hepatitis C 2018. J Hepatol. 2018;69:461-511.

16. Toyoda H, Kumada T, Takaguchi K, Shimada N, Tanaka J. Changes in hepatitis c virus genotype distribution in Japan. Epidemiol Infect. 2014;142: 2624-8.

17. Drafting Committee for Hepatitis Management Guidelines, the Japan Society of Hepatology. Japan Society of Hepatology guidelines for the management of hepatitis C virus infection: 2019 update. Hepatol Res. 2020;50:791-816.

18. Asahina Y, Itoh Y, Ueno Y, et al. Ledipasvir-sofosbuvir for treating Japanese patients with chronic hepatitis $\mathrm{C}$ virus genotype 2 infection. Liver Int. 2018;38:1552-61.

19. Ohno O, Mizokami M, Wu RR, et al. New Hepatitis $\mathrm{C}$ Virus (HCV) genotyping system that allows for 
identification of HCV genotypes $1 \mathrm{a}, 1 \mathrm{~b}, 2 \mathrm{a}, 2 \mathrm{~b}, 3 \mathrm{a}$, 3b, 4, 5a, and 6a. J ClinMicrobiol. 1997;35:201-7.

20. Sterling RK, Lissen E, Clumeck N, APRICOT Clinical Investigators, et al. Development of a simple noninvasive index to predict significant fibrosis in patients with HIV/HCVcoinfection. Hepatology. 2006;43:1317-25.

21. The French METAVIR Cooperative Study Group. Intraobserver and interobserver variations in liver biopsy interpretation in patients with chronic hepatitis C. Hepatology. 1994;20:15-20.

22. Vallet-Pichard A, Mallet V, Nalpas B, et al. FIB-4: an inexpensive and accurate marker of fibrosis in HCV infection: comparison with liver biopsy and fibrotest. Hepatology. 2007;46:32-6.

23. Matsuo S, Imai E, Horio M, Collaborators developing the Japanese equation for estimated GFR, et al. Revised equations for estimated GFR from serum creatinine in Japan. Am J Kidney Dis. 2009;53: 982-92.

24. Kidney Disease: Improving Global Outcomes (KDIGO) CKD Work Grouop. CKDIGO 2012 linical practice guideline for the evaluation and management of chronic kidney disease. Kidney Int. 2013;3(3):1-150.

25. Kanda Y. Investigation of the freely available easyto-use software 'EZR' for medical statistics. Bone Marrow Transplant. 2013;48:452-8.

26. Lawitz E, Mangia A, Wyles D, et al. Sofosbuvir for previously untreated chronic hepatitis C infection. N Engl J Med. 2013;368:1878-87.

27. Jacobson IM, Gordon SC, Kowdley KV, POSITRON Study; FUSION Study, et al. Sofosbuvir for hepatitis C genotype 2 or 3 in patients without treatment options. N Engl J Med. 2013;368:1867-77.

28. Zeuzem S, Dusheiko GM, Salupere RM, VALENCE Investigators, et al. Sofosbuvir and ribavirin in HCV genotypes 2 and 3. N Engl J Med. 2014;370: 193-201.
29. Koff RS. Review article: the efficacy and safety of sofosbuvir, a novel, oral nucleotide NS5B polymerase inhibitor, in the treatment of chronic hepatitis $\mathrm{C}$ virus infection. Aliment PharmacolTher. 2014;39:478-87.

30. Le TK, Macaulay D, Kalsekar A, et al. Costs and resource utilization associated with anemia and rash in chronic hepatitis c patients treated with direct-acting antiviral agents in the United States. ClinTher. 2015;37:1713-25.

31. Gane EJ, Hyland RH, Yang Y, et al. Efficacy of Ledipasvir Plus Sofosbuvir for 8 or 12 weeks in patients with hepatitis $C$ virus genotype 2 infection. Gastroenterology. 2017;152:1366-71.

32. Liu CJ, Chuang WL, Sheen IS, et al. Efficacy of Ledipasvir and Sofosbuvir treatment of HCV infection in patients coinfected with HBV. Gastroenterology. 2018;154:989-97.

33. Liu CH, Su TH, Liu CJ, et al. Sofosbuvir-based direct acting antiviral therapies for patients with hepatitis $\mathrm{C}$ virus genotype 2 infection. J GastroenterolHepatol. $2019 ; 34: 1620-5$.

34. Ogawa E, Nomura H, Nakamuta M, Kyushu University Liver Disease Study (KULDS) Group, et al. Ledipasvir and sofosbuvir for 12 weeks for hepatitis $C$ virus genotype 2 infection: a propensity score matched analysis. Hepatol Res. 2020;50: 174-81.

35. US Food and Drug Administration, Center for Drug Evaluation and Research. Virology review, sofosbuvir and ledipasvir. 2020. https://www.accessdata. fda.gov/drugsatfda_docs/nda/2014/ 205834Orig1s000MicroR.pdf. Accessed 15 June 2020.

36. Welzel TM, Bhardwaj N, Hedskog C, et al. Global epidemiology of HCV subtypes and resistance-associated substitutions evaluated by sequencingbased subtype analyses. J Hepatol. 2017;67:224-36.

37. Chung H, Ueda T, Kudo M. Changing trends in hepatitis $C$ infection over the past 50 years in Japan. Intervirology. 2010;53:39-43. 